\title{
Towards Data-Driven Culture in a Spanish Automobile Manufacturer: A Case Study
}

\author{
Maria Esteller-Cucala ${ }^{1,2}$ iD, Vicenc Fernandez ${ }^{1}$ iD, Diego Villuendas ${ }^{2}$ iD \\ ${ }^{1}$ TechTalent-Lab, Department of Management, Universitat Politècnica de Catalunya · BarcelonaTech (Spain) \\ ${ }^{2}$ SEAT, S.A. (Spain) \\ maria.esteller.cucala@gmail.com,vicenc.fernandez@upc.edu,Diego.Villuendas@seat.es
}

Received: October 2019

Accepted: February 2020

\begin{abstract}
:
Purpose: Data-driven decision-making is a growing trend that lots of companies are nowadays willing to adopt. However, the organizational transformation needed is not always as simple and logical as it could seem and the comfort of the old habits can dim the change effort. The purpose of this study is to identify the potential problems that may arise in a real company's transformation from a traditional intuition-driven decision-making model to a data-driven model.
\end{abstract}

Design/methodology/approach: In order to reach this goal, a single case study method was used. Initially a literature review was conducted to analyze both the importance of the change to a data-driven culture and the process of organizational change. Thus, a case study method was adopted in a company of the automotive sector that included experimentation in the website design decision-making process.

Findings: As a result of the case study, it was found that all the most cited risks for the organizational change process commented in the literature appeared in the project. However, even being warned of potential dangers the specific actions to prevent the damages were not trivial.

Originality/value: The study presents in detail, the application of an organizational change model in a company. Important insights can be extracted from the specific case of a digitalization performed inside traditional industrial company.

Keywords: organizational change, digital transformation, digitalization, data-driven transformation, website testing

\section{To cite this article:}

Esteller-Cucala, M., Fernandez, V., \& Villuendas, D. (2020). Towards Data-Driven Culture in a Spanish Automobile Manufacturer: A Case Study. Journal of Industrial Engineering and Management, 13(2), $228-245$. https://doi.org/10.3926/jiem.3042

\section{Introduction}

In the nowadays fast-changing world, companies are successfully only if they adapt their efficiency and speed to the increasingly active business environment (Christopher, 2011; Wassner \& Brebion, 2018). Therewith, organizations have realized the criticality of digitalization for their own business (Kohnke, 2016). Currently, digitalization (also known as digital transformation) is broadly understood through four main areas or technologies: analytics, mobile, 
cloud and social media technologies (Cray, 2014). In this work we are focusing the first one (analytics). This area of the digital transformation allows innovative forms of information processing for better insights and decision making. Subject-matter experts cannot always assimilate the changing reality fast enough to be considered the only source of truth (Olsson \& Bosch, 2014). Therefore, the traditional experience- or intuition-driven methods to guide decision making are no longer sufficient (Condo \& Seguin, 2019). For this reason, instead of basing decision on expertise, intuition or even opinion, the trend in software development is to start making decisions based on data, and specifically, data coming from user experimentation (Olsson, Bosch \& Fabijan, 2017).

In almost any field, observations and experience can lead to theories or hypotheses about what is making it work or fail for the user, however experimentation is required to demonstrate if these theories and hypotheses are correct (Montgomery, 2001). In the specific case of website development, one of the most generalized ways of achieving this data-driven user-feedback experimentation is by using online controlled experiments (known in its simplest version as A/B testing) (Fabijan, Dmitriev, McFarland, Vermeer, Holmström Olsson \& Bosch, 2018). With the adoption of online controlled experiments, proposed changes or questions should always be structured and treated as experiments. With it, the decision-making switches from a battle over arguments or opinions around the table to a scientific, data-driven process directly based on users (Crook, Frasca, Kohavi \& Longbotham, 2009; Fabijan, Dmitriev, Holmstrom-Olsson \& Bosch, 2018; Kohavi, Henne \& Sommerfield, 2007; Spear, 2004).

Data-driven companies (e.g. Google, Microsoft or Netflix), commonly use website experiments in order to gather the data needed to make decisions about whether a website change should be implemented and launched to all the users (Tang, Agarwal, O’Brien \& Meyer, 2010). The importance of website experimentation is even more visible when some of those big digital companies report that $60-90 \%$ of their experiments do not show positive results. This means that, what they hypothesized as having value for their users was in fact wrong, and, in some cases, it could have even damaged the website performance or user experience in case of being directly launched without the experiment (Kaushik, 2006; Kohavi, Deng, Longbotham \& Xu, 2014; Rissanen \& Munch, 2015).

Including a few online experiments in organizations decision-making processes can provide great insights into how users are using a feature. Nevertheless, the real impact on the company comes with running frequent experiments and using experimental results as major input to drive decisions (Fabijan, Dmitriev, Olsson \& Bosch, 2017; Kohavi et al., 2007). However, this requires organizational changes that instill experimentation in organizations culture and practice, and this transformation is far from intuitive (Fabijan, Dmitriev, McFarland, et al., 2018). The transformation needed to switch from experience-driven to data-driven decision-making is not simple, especially for industrial organizations, more familiar with traditional manufacturing processes (Fabijan, Dmitriev, McFarland, et al., 2018). Therefore, the number of companies that successfully transform into data-driven stays low (Fabijan et al., 2017). The difficulties and also the high failure rate of this kind of organizational transformation has been widely discussed in the literature, also lists of different critical success/failure factors and possible solutions (or mitigations) have been studied and presented (Aslam, Muqadas, Imran \& Saboor, 2018; Fui-Hoon Nah, Lee-Shang Lau \& Kuang, 2001; Vakola \& Wilson, 2004). However, authors and practitioners keep reporting unsuccessful cases (Ainul-Azyan, Pulakanam \& Pons, 2017; Imran, Rehman, Aslam \& Bilal, 2016). Given this contradiction, some questions may arise, such as; are the organizational change success/failure factors manageable by the organizations to control the results of the transformations? Is there anything that companies should do to overcome given failure factors in their organizations? Are companies aware of the research previously done about organizational change success factors? Are companies planning and developing their organizational change processes according to the conclusions previously brought out by researchers' publications? On these bases, this study seeks to identify the potential problems that may arise in a real company's transformation from a traditional intuition-driven decision-making model to a data-driven model once the company has been forewarned of the main factors associated with failed change initiatives.

To achieve this goal, this study presents a case study of how moving from traditional to data-driven decision-making can be applied in the case company, specifically for the website development. Based on the results of a literature review, the case study reflects the differences between theoretical risks and its impact on a real organizational change process. 
While studies applying those kind of transformation exist (Neely \& Stolt, 2013; Rissanen \& Munch, 2015), most of them are based on software companies. The relevance of this specific case is that, first, even if it is based in the website team transformation, is held in a traditional industrial company with rooted processes and culture that could mean a handicap for the digitalization. Second, the organizational change of this case company is planned and developed taking into account a list of previous studied and published success/failure factors organizational transformations.

\section{Organizational Change Literature Review}

As previously stated, a real organizational transformation is needed in order to become truly data-driven. Given that this transformation is almost necessary for the organization to adapt to the new digital environment (Fabijan et al., 2017), this transformation can be addressed in the company as an incremental organizational change (Cao, Clarke \& Lehaney, 2000).

Organizational change processes have different phases (Imran et al., 2016). The processes are highly context dependent (Hughes, 2011) and might be far from intuitive (Imran et al., 2016). Even if there is no agreement on the specific failure rate of organizational change (Cândido \& Santos, 2015; Hughes, 2011), authors agree in that fail rate is not depreciable (Kotter, 2006) and the difficulties of achieving organizational changes should not be trivialized (Attaran, 2000).

Lewin's organizational change model is one of the first known models and also one of the most extended ones (Elrod \& Tippett, 2002). This simplistic model (Lewin, 1951) is based in three phases to describe change: Unfreeze, Move/Change and Freeze. For a long time, from Lewin's model first presentation (Rashford \& Coghlan, 1989) and especially since the mid 80 s, a wave of practitioners presented their organizational change models or approaches (Johansson \& Heide, 2008). Some of the most well-known ones are Bullock and Batten's four-phase model (Bullock \& Batten, 1985), Kanter's ten commandments for executing change (Kanter, Stein \& Jick, 1992) and Kotter's eight-stage model on how to manage change successfully (Kotter \& Rathgeber, 2005; Kotter, 1996). However, these three models harmonize with the original idea of the unfreeze-change-freeze model, like most of the models presented after Lewin's one (Elrod \& Tippett, 2002; Erwin \& Garman, 2010; Johansson \& Heide, 2008). Considering the vast amount of existing organizational change models in the literature (Elrod \& Tippett, 2002) and stated that the change process is not easy, the selection of approach and also its application in the particular organization are key in the success of the transformation (Imran et al., 2016).

Nevertheless, the application of a change model does not ensure the success of the organizational transformation. Various authors have presented a wide variety of causes of failures of organizational change efforts (Appelbaum, Cameron, Ensink, Hazarika, Attir, Ezzedine et al., 2017; Aslam et al., 2018). Their studies have shown that most of the causes have strong correlations between them. After conducting a literature review on the topic or organizational change with special focus on change success (and failure) causes, we identified seven causes as being given special focus or recurrence in the literature. These seven causes of organizational change failure frequently commented and studied in the literature are:

1. Not following an organizational change procedure. As previously introduced, organizational transformations are not easy processes and there is no shortage of authors reporting unsuccessful cases (Aslam et al., 2018). To have better chances of success a methodology should be well-defined, alienated with the business structure and executed properly (Attaran, 2000; Brisson-Banks, 2010; Rajan \& Ganesan, 2017). Developing an instruction plan or roadmap of the change process beforehand is considered a critical factor for the success of the transformation (Chrusciel \& Field, 2006). This is, not only to have a management strategy, but to have an entire organizational change model/process to follow (Appelbaum et al., 2017; Galli, 2019).

2. Not filling the knowledge gap. No matter how complete is the organizational change strategy and how many details have been considered, transaction to a continuous experimentation model can make the stakeholders feel uncomfortable and scary (Neely \& Stolt, 2013). When there is a transformation within an organization, a gap of knowledge is generated, positions and roles might be changed and new abilities 
might be needed. Specifically, in the case of a change in the decision-making process, it can produce in the team members the shared belief that the team is not safe and this could be considered as part of the emotional response to change (Erwin \& Garman, 2010). For example, traditionally, companies have considered the ideation and development of new versions of the website (or other products) as a success. Therefore, the transformation into testing each new idea and creating the possibility of proving it wrong may generate psychological unsafety within the team. It is imperative for the success of the transformation to make teams be prepared in learning from the experimentation, accept that most ideas will fail and to be constantly thinking in what to try next (Fabijan, Dmitriev, McFarland, et al., 2018). Filling the knowledge gap is mainly solved by providing training (Imran et al., 2016; Vakola \& Wilson, 2004). Users should effectively be trained and provided with documentation in order for them to be able to not only adopt the transformation but also understand, support and keep it after the change process (Wong, Chau, Scarbrough \& Davison, 2005). This is considered a crucial aspect for any digital transformation, where building digital capabilities is part of the change (Kohnke, 2016). Moreover, filling the knowledge gap not only means providing proper training. It also means giving users support at all stages of the change process, monitoring the level of understanding of the change initiative and creating an organizational knowledge sharing culture able to foster idea generation, organizational learning, competence and innovation (Aslam et al., 2018; Attaran, 2000).

3. Failure to cope with people's resistance to change. Human-related factors and the employee's response to change are two of the core topics to take care of when planning an organizational change (Erwin \& Garman, 2010) and, accordingly, the most studied ones (Hamdi \& Abouabdellah, 2018). Resistance to change, change cynicism or change anxiety should be some of the main concerns when it comes to organizational change initiatives, as they could slow down or even terminate the change efforts (Appelbaum, Profka, Depta \& Petrynski, 2018; Attaran, 2000; Erwin \& Garman, 2010; Imran et al., 2016; Vakola \& Wilson, 2004). Behaviors in response to change can be labeled supportive or resistant, active or passive and covert or overt (Bovey \& Hede, 2001). But also, resistance to change has been found to be a multi-dimensional response including behavioral, cognitive and emotional dimensions (Piderit, 2000), what makes it a complex concept to be taken care of (Attaran, 2000; Erwin \& Garman, 2010). This failure cause is strongly correlated with the previous one (knowledge gap filling) as far as the lack of training might give employees fear of being unable to perform in the new way, producing this negative response to change (Martin, Jones \& Callan, 2005). On the contrary, self-efficacy (feeling able to perform) and perception of personal gain might rise employee's willingness to welcome change (Aslam et al., 2018; Chrusciel \& Field, 2006; Martin et al., 2005). Moreover, employees that perceive an alignment of the organization's vision and the change proposal tend to express lower resistance to change (Appelbaum et al., 2017). As further explained in the following paragraphs, other factors (i.e. change readiness and management involvement) are correlated with employees' resistance to change.

4. Lack of sense of urgency. A remarkable proportion of the organizational change models described in the literature (and especially the ones based on Lewin's model) start with an unfreeze phase (Elrod \& Tippett, 2002; Erwin \& Garman, 2010; Johansson \& Heide, 2008; Kotter \& Rathgeber, 2005). This phase mainly consists in generating sense of urgency in the organization, what could be considered equivalent to motivation for the change and intend to be prepared for the change (Martin et al., 2005; Ouedraogo \& Ouakouak, 2018). It is not only important to transmit the sense of urgency to the employees, but also to the management involved in the change (Antony \& Banuelas, 2002; Kotter, 2006). This motivational attitudes and determination to succeed need to be present in all the participants of the change from the first planning phases and during the entire transformation process (Soja, 2016). A lack of sense of urgency is considered as one of the primary causes of failure of organizational change efforts (Aslam et al., 2018) and is specifically significant and challenging for digital transformations (Kohnke, 2016).

5. Insufficient organizational readiness for change. According to previous authors, another factor related to the unfreezing phase and widely commented in the literature is the readiness for change (Aslam et al., 
2018). The readiness for change englobes the positive thoughts, attitudes and intentions regarding change and the beliefs about the need for change, its benefits and its implications (Aslam et al., 2018; Imran et al., 2016). Although readiness for change might seem similar to the previous factor (sense of urgency or motivation), it has more to do with the previous organization culture: continuous attention to employees psychological responses, information sharing, trust and teamwork (Brisson-Banks, 2010; Kohnke, 2016). The organization structure or company's condition will play a key role in the company's readiness change and, thus, in the change success (Antony \& Banuelas, 2002; Soja, 2016). Flat (horizontal) organizations are more used to open communication channels and facilitate participation in decision-making, easing the organizational transformation processes (Vakola \& Wilson, 2004). Moreover, the culture of contiguous innovation and internal collaboration of some organization are critical drivers of transformation success (Long, Looijen \& Blok, 2018). Finally, the staff perception of organizational readiness to cope with change can be considered to have even more effect on the transformation result than the actual organizational readiness for change, being critical success factor (Chrusciel \& Field, 2006).

6. Insufficient management support and involvement. Management involvement in the process is key for the success of the transformation effort (Aslam et al., 2018; Attaran, 2000; Wong et al., 2005). This is not only due to the influential power of the management itself but due to how management involvement induces employees' positive attitudes towards change and higher levels of commitment to the transformation process (Martin et al., 2005). On the one hand, the actual management support (i.e. resources dedicated, communication efforts, empowering of the leading) as well as management involvement (stakeholders conflicts resolution, task prioritization and monitoring, internal leadership through the process) are critical factors to be taken care of (Alsulami, Rahim \& Scheepers, 2013; Kohnke, 2016). On the other hand, part of the effect of the management actions lie in how they are perceived by employees (Appelbaum et al., 2017). Employees who perceive enthusiastic and coherent vision for the organizational change in their leaders (or managers) tend to be more positive, committed and have less resistance to change (Kohnke, 2016; Martin et al., 2005). Furthermore, consistency of management actions with the proposal, drives organizational trust (Erwin \& Garman, 2010). Consequently, this management support needs to be visible, consistent, overt and continued (Venugopal \& Suryaprakasa-Rao, 2011). Finally, in the specific case of digital transformations, even if the need to turn into a data-driven company may be motivating and empowering, the associated cost of the organizational change (i.e. teams' time and effort, temporal loss of productivity) might undermine the initiative. In this case, authors emphasize the importance of this risk and the importance for managers to have the ability to also legitimate the transformation proposal plan in terms of time and money to avoid employees from self-efficacy loss fear (Neely \& Stolt, 2013).

7. Lack or infectivity of communication. The role of communication in change processes has been one of the most commented factors in the literature (Johansson \& Heide, 2008). First of all, the communication culture of the organization (i.e. communication with immediate supervisors, communication frequency and information sharing) will have an effect on the transformation directly and indirectly (by affecting for example commitment to change, readiness for change and job satisfaction) (Attaran, 2000; Martin et al., 2005). Secondly, communication about the change is necessary for all the involved employees to understand and share the goal, vision and processes of the transformation (Ouedraogo \& Ouakouak, 2018; Venugopal \& Suryaprakasa-Rao, 2011). But also, constant and effective communication efforts affect employees commitment to change, trust and perception of situational control (Appelbaum et al., 2017; Martin et al., 2005; Vakola \& Wilson, 2004). Finally, enabling bidirectional communication (empowering employees input and feedback) promotes acceptance of the change, gives employees a sense that they can actively contribute to the change and increases productivity (BrissonBanks, 2010; Martin et al., 2005). Given the importance of communication in the final result of organizational changes, some studies are analyzing the effect of new communication channels (i.e. social media) and extensive communications (i.e. creating general awareness of the importance of digitalization) (Aslam et al., 2018; Kohnke, 2016). 
During the review of the literature, other factors for the organizational change success (or failure) have been widely studied and commented. For example, the extent and turbulence of the change (both in how big is the change and how many people is involved) are commented factors in the literature (Antony \& Banuelas, 2002; Appelbaum et al., 2017). Also the external influences or events (like suppliers attitudes or governmental regulations) have been reported to have major impact on the transformation process outcome (Antony \& Banuelas, 2002; Long et al., 2018). Moreover, internal actions such as to have a monitorization system to track the transformation results and to determine anchoring points has been commented to have extensive impact in the change results (Antony \& Banuelas, 2002; Fui-Hoon Nah et al., 2001; Soja, 2016). However, the amount of research addressing the seven causes previously listed is noticeably more frequent.

Once the main factors associated with failed organizational change efforts have been reviewed and stated that an organizational transformation is a highly context dependent process, this study seeks to identify the potential problems that may arise in a real company's transformation from a traditional intuition-drive decision-making model to a data-driven model. To this effect, the research question (RQ) of this study is:

- Which are the potential problems that may arise in a real company's transformation from a traditional intuition-drive decision-making model to a data-driven model known beforehand the main factors associated with failed organizational change?

\section{Methodology}

In order to answer the former RQ we suggest using a case study approach (Yin, 2002). Case studies are especially useful to provide greater familiarity with the problem, to make it more explicit when the boundaries between the phenomenon and its context are not clearly evident (Yin, 2003). As we want to explore a situation in which the set of outcomes is not clear, the specific method used is an exploratory case study (Baxter \& Jack, 2008).

After a first analysis of the problem based on the literature review and the selection of the case study approach, the case selection criterion was a traditional company in the digitization process, specifically in the main area of analytics and decision making. Even if digitalization has the potential to improve analysis for decision making, it remains hard for organizations to integrate it into their day-to-day decision making (Cray, 2014). The organization analyzed in this case study was, at the time of the study, planning the transformation into data-driven decisionmaking for the digital platforms' involved teams (websites teams).

The selected organization for this case study is an automotive brand, specifically the case study is based on the organizational transformation of the decision-making culture within the digital platforms' team. While being a digital team related to software products, the industrial organizational context of this department has played a key role in the processes and decision-making procedures for the development of the website. Lately, the organization is performing its digitization process (Kohnke, 2016) with a set of different initiatives and approaches. Under this digitalization framework, the objective of the studied project is to make the necessary organizational change to switch from a traditional intuition-driven decision-making model to a data-driven model. The project is still ongoing (at the time of this study), the duration has been so far one and a half years and all the teams related to the planning, design, development, operation and use of the organization's external websites (excluding internal HR websites and intranets) are involved.

Once the case was selected, as planning for the research, a protocol was developed to conduct the case study (containing details about scope of the paper, study objects, profile of interview respondents, data collection and analysis, etc.). The data collection was comprised using four of the six main case study sources of evidence for case studies research (Yin, 2003). Used data sources are presented in Table 1.

Finally, the results were analyzed and compared with the literature previously reviewed in order to extract and report conclusions. Figure 1 presents the steps of the methodological procedure for conducting this research. 


\begin{tabular}{|l|l|}
\hline Data collection procedure & Comments \\
\hline Participant-observation & $\begin{array}{l}\text { During the time of the study two of the researchers were employees } \\
\text { in the company, one in a managerial role and the other one directly } \\
\text { involved in the case study project. With it, action research was } \\
\text { employed in the data collection. }\end{array}$ \\
\hline Unstructured interviews & $\begin{array}{l}\text { In order to gather more depth and detail of the employees' } \\
\text { perception and thought, non-directive interviews were selected. }\end{array}$ \\
\hline Project documentation & $\begin{array}{l}\text { The analyzed documentation includes both the documents } \\
\text { generated within the scope of the project and the materials used for } \\
\text { organizational communication. }\end{array}$ \\
\hline Meeting minutes and email record communications & $\begin{array}{l}\text { This written information was collected in order to track the formal } \\
\text { project progress. }\end{array}$ \\
\hline
\end{tabular}

Table 1. Data collection procedures

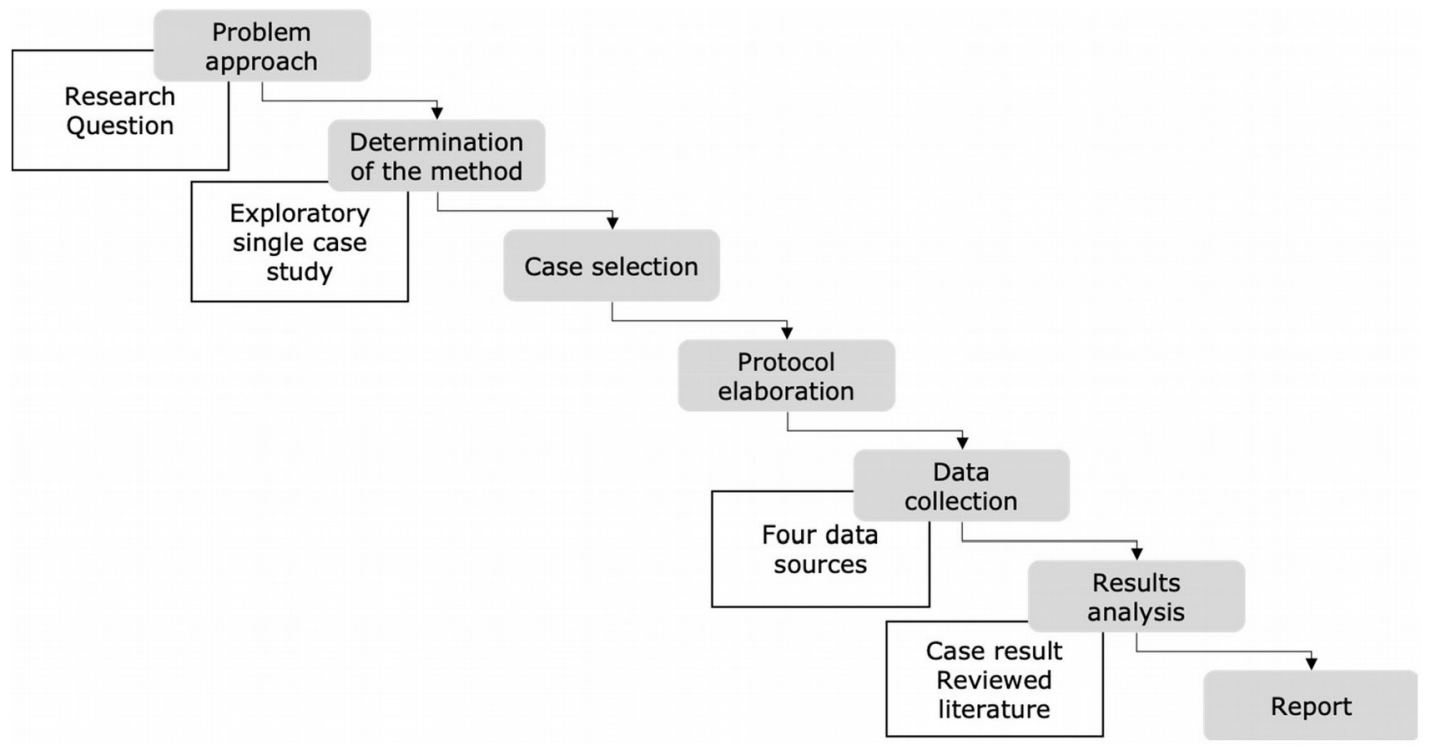

Figure 1. Methodological procedure followed in this study

\section{Description of the Case}

As previously described, the context of the case is the digital platforms team inside an automotive brand organization. The participants involved in this project can be classified in different groups, each of them including from management roles to general workforce employees. In this organization, the stakeholders' landscape is organized around two main products: the master website (a template of the website on the basis of which market local teams adapt the content to generate their websites) and the customer websites (all the final websites that users can access). There is only one master website and more than 40 customer websites. The approximate diagram of the organization teams is presented in Figure 2.

With such a complex and extensive network of collaborations and communications the organizational change needed to transform the decision making from intuition-driven to data-driven was considered a complex transformation. In order to maximize the chance of success, the list of 7 of the most commented causes for organizational change failure in the literature (see Organizational change literature review) was considered before starting the transformation planning.

The first decision of the planning, according to the first item of the list of failure causes (not following an organizational change procedure), was to stick to an organizational change model process. The starting consideration was to use Lewin's 3 step framework (Lewin, 1951) because it was considered simple and the principles behind it are understandable for the team. However, the team was not able to find with that model a 
practical guidance on what to do. Therefore, they decided to go for a model with more business-oriented steps and, if possible, based on Lewin's principles. In the literature, there is no shortage of organizational change models based on Lewin's unfreeze-move-freeze model (Elrod \& Tippett, 2002). The additional selection criteria for the model were to be top-drown (because of the nature of the project), to be valid for small project scopes (because this change is not affecting the entire organization but only the website related departments) and to have special focus on communication and team involvement (according to the list of failure causes) (Galli, 2019). Finally, the chosen framework was the Kotter's eight-stage model (Kotter \& Rathgeber, 2005; Kotter, 1996).

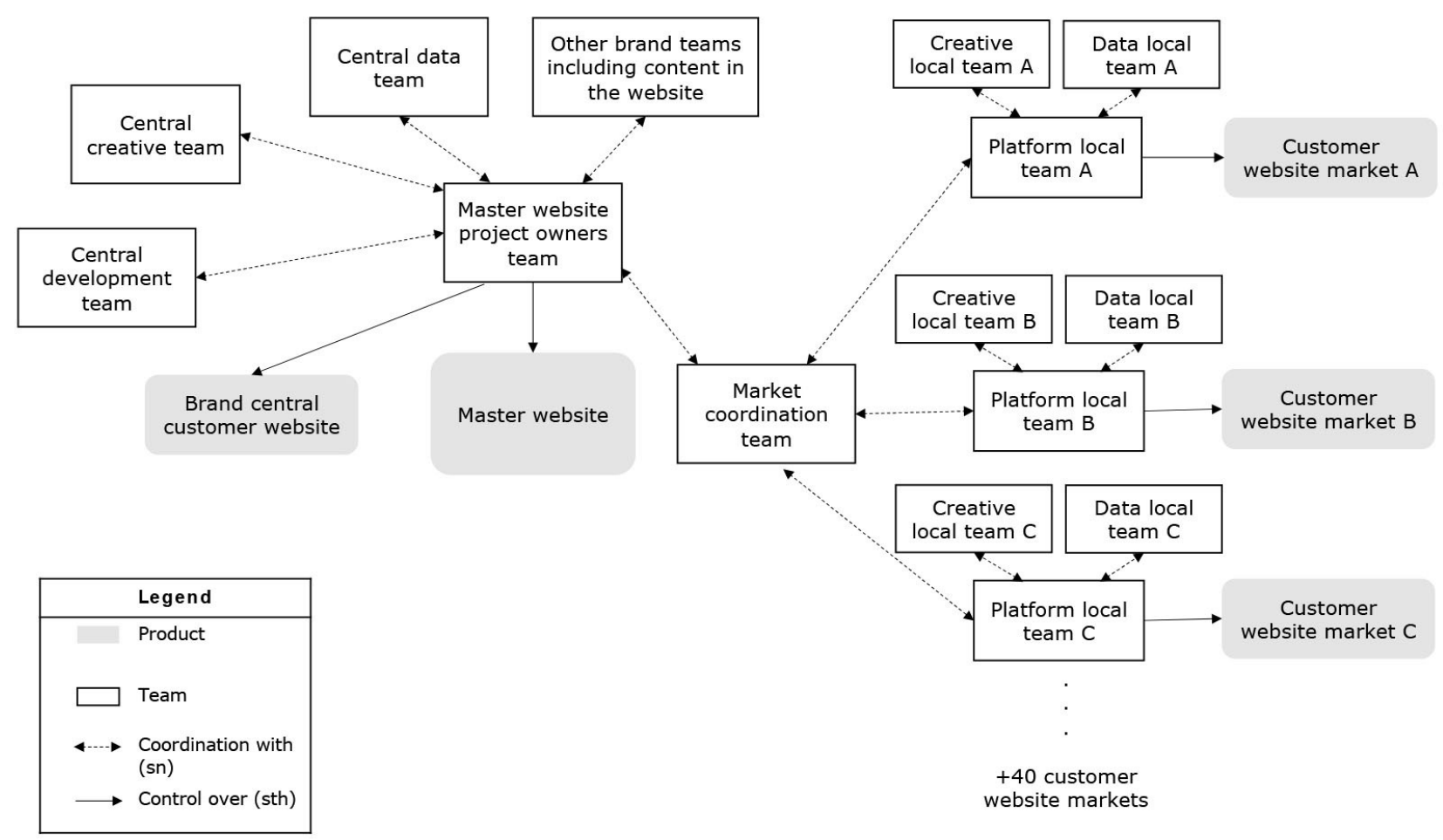

Figure 2. Approximate diagram of the organization sub-teams

Once the model was selected, the organization strictly followed the Kotter and Rathgeber's eight steps of the model. Table 2 summarizes the organizational change performed in this project.

\begin{tabular}{|l|l|}
\hline Step & Practical implementation \\
\hline 1.Establish a sense of urgency & $\begin{array}{l}\text { The need for change was presented with three main arguments: } \\
\text { - Website experimentation was already a reality in other companies } \\
\text { - Intuition or expertise driven decisions were one of the main causes of discussion } \\
\text { between teams } \\
\text { Website experimentation brings the opportunity to include the user in the } \\
\text { decision making }\end{array}$ \\
\hline 2.Form a powerful guiding coalition & $\begin{array}{l}\text { A specific group was created and supported by direct management. This group } \\
\text { included members from project-owners, creative and data central teams. }\end{array}$ \\
\hline 3.Create a vision & $\begin{array}{l}\text { The shared vision was based on driving the decisions on users' data, collaboration } \\
\text { between team and transparency. }\end{array}$ \\
\hline 4.Communicate the vision & $\begin{array}{l}\text { Documentation and training were shared to all the involved teams. } \\
\text { The guiding team communicated their willingness to train, support and solve } \\
\text { questions. }\end{array}$ \\
\hline
\end{tabular}




\begin{tabular}{|l|l|}
\hline Step & Practical implementation \\
\hline 5.Empower others to act on the vision & $\begin{array}{l}\text { Non-technical training guides and templates were created for the generation of } \\
\text { hypothesis, test plans, understanding of the results and most common vocabulary. } \\
\text { Also, the processes were designed considering the autonomy of local teams and the } \\
\text { testing tool access was rolled out to all teams who required it. }\end{array}$ \\
\hline 6.Plan for and create short term wins & $\begin{array}{l}\text { During the second month of the project first experiments were created leaded by } \\
\text { the guiding teams and results were extensively shared and explained. }\end{array}$ \\
\hline $\begin{array}{l}\text { 7.Consolidate improvements and } \\
\text { produce more change }\end{array}$ & $\begin{array}{l}\text { Three main actions were performed: } \\
\text { Extending the first experiments to other markets } \\
\text { - Generating first website developments bases on tests results } \\
\text { - Fortnightly global communication sharing experiment results }\end{array}$ \\
\hline 8.Institutionalize new approaches & The company is working in the follow-up strategy. \\
\hline
\end{tabular}

Table 2. Organizational change process followed

\section{Results and Discussion}

After compiling and reviewing the information from the different sources commented in the methodology (see Methodology), we evaluate the main results obtained for each step of the change process. The risks exposed in the literature review (see Organizational change literature review) are presented considering the risks predicted to appear in each step (based on the literature), the actions taken in order to minimize each risk (not all the risks predicted were specifically associated with mitigation actions), also the actually detected risks are commented.

Step 0. Before starting the first step:

a. Predicted risks and mitigation actions taken in each case:

- Not following an organizational change procedure (Appelbaum et al., 2017). As previously introduced, the company decided to follow Kotter's eight-stage model (Kotter, 1996) step by step.

- Insufficient organizational readiness for change. The complexity of the structure of teams, their international physical locations, linguistic barrier and different skilled roles were considered as barriers for the readiness for change (Aslam et al., 2018; Imran et al., 2016). No actions were taken in this regard because it was considered to be something rooted in the organization and its structure and to be solved with the change itself.

Step 1. Establish a sense of urgency:

b. Predicted risks and mitigation actions taken in each case:

- Lack of sense of urgency. Some teams were already experimenting in their local websites, whilst other teams were established in the old decision-making culture (Aslam et al., 2018). This risk is considered to be specially significant for digital transformations (Kohnke, 2016). Some actions were taken in order to equalize the different teams' motivation and sense of urgency (i.e. the organizational change initiative was presented to the teams in an event where they were all physically together, instead of the individualized and telematic communications).

c. Detected risks:

- Despite the predictions, no risks were clearly detected in this stage of the project.

Step 2. Form a powerful guiding coalition:

a. Predicted risks and mitigation actions taken in each case:

- Insufficient organizational readiness for change. The vertical structure of the organization was considered a potential risk for the teamwork of the guiding coalition (Kotter, 2006; Vakola \& Wilson, 2004). No actions were taken to mitigate this predicted risk. 
- Insufficient management support. In order for the guiding coalition to have any change of being followed, local teams needed to perceive the coalition as supported by management (Appelbaum et al., 2017; Attaran, 2000). In order to minimize this risk, the management was completely supportive with the project and the guiding coalition. To make this support visible for the rest of the teams, the first presentation of the organizational change process was introduced by a manager and the guiding coalition kick-off-meeting was also led by managers of the different teams.

b. Detected risks:

- With the beforehand perditions and the mitigation actions taken, none of the predicted risks were detected to have any effect on the change process at this step. On the one hand, the management showed and agreed the vision of the change initiative and the guiding coalition had not teamwork conflicts. On the other hand, no lack of management support was detected. Moreover, no other risks were detected to have an effect.

Step 3. Create a vision:

a. Predicted risks and mitigation actions taken in each case:

- Failure to cope with people's resistance to change. If the vision is perceived as too complicated, too vague or not aligned enough with the general vision of the company teams' members my express negative responses to the change (Appelbaum et al., 2017; Kotter, 2006). No actions were taken to mitigate this risk.

b. Detected risks:

- The vision was perceived as alienated and the theoretical proposal seemed to be welcomed among most teams' members. And no risks were detected to have an effect on this stage.

Step 4. Communicate the vision:

a. Predicted risks and mitigation actions taken in each case:

- Not filling the knowledge gap. As a digital transformation, one of the main risks to be considered was not only the lack of experimentation skills (Kohnke, 2016), but also the individual perception of this knowledge gap (because this could lead to resistance to change) (Martin et al., 2005). Considering this possible risk, the vision was communicated in a simple way so that every team could understand and share the idea behind the organizational change initiative.

- Lack or infectivity of communication. The goal of communication at this step was to be credible and abundant (Kotter, 2006), to mobilize the organization for the change (Kohnke, 2016) and to encourage feedback and share the information needed in order for all the involved teams to feel part of the change (Appelbaum et al., 2017; Brisson-Banks, 2010). There was special focus on communicating the initiative to all the involved teams and to make it simple enough for them to understand.

b. Detected risks:

- As a result of the extensive communication and the focus on simplifying the concepts related to the new vision, the initiative seemed to be understood and supported by some teams. However, first signals of resistance to change were found.

Step 5. Empower other to act on the vision:

a. Predicted risks and mitigation actions taken in each case:

- Not filling the knowledge gap. When teams were encouraged to start experimenting by themselves, one of the main risks was them not filling prepared or autonomous (Wong et al., 2005). Accordingly, specific documentation was generated and shared with the teams, also the processes were designed to 
include both autonomous skilled teams and non-autonomous teams needing support. Finally, the willingness of the guiding coalition to support other teams was emphasized in all the communications.

- Failure to cope with people's resistance to change. The possibility to test ideas and prove them wrong may generate psychological unsafety within employees (Fabijan, Dmitriev, McFarland, et al., 2018). At this point the proactive proposal of experiments was numerous by the most autonomous local teams but it was scant by the rest of the teams.

b. Detected risks:

- Because of the lack of experience on website testing, some teams started generating experiment proposals that were based on small changes visible only for small numbers of visitors. Due to the small number of visitors and the slightly perceptible changes, the tests ended up with no significant results, what lowered the motivation on the new vision.

Step 6. Plan for and create short term wins:

a. Predicted risks and mitigation actions taken in each case:

- Lack of sense of urgency (motivation). Although presenting short term wins is important and necessary for the success of the change initiative (Kotter, 2006), short term wins do not have the same impact as the whole change process. Therefore, if short term wins do not cope with the high expectations generated during the communication of the vision, the motivation might decrease and the change might be abandoned beforehand (Martin et al., 2005; Ouedraogo \& Ouakouak, 2018; Venugopal \& Suryaprakasa-Rao, 2011).

- Lack or infectivity of communication. In connection with the previous point, both overcommunicating and under-communicating the short-term wins could have a negative impact on the motivation, thus, on the final results of the change efforts (Appelbaum et al., 2017; Martin et al., 2005; Vakola \& Wilson, 2004). Short term wins were extensively communicated and well received by all the teams involved.

b. Detected risks:

- Insufficient management support and involvement. After the first short term wins, there was a risk that management lose interest on the initiative (they might consider it finished or controlled). This risk was not predicted based on the literature, but it was detected during the project observation and interviewing the guiding team members. Management support was maintained but management involvement was significantly decreased, as far as the initiative vision was already shared and the guiding coalition was perceived as strong enough, this was not detected to have any major impact on employees' motivation nor on the initiative development.

Step 7. Consolidate improvements and produce more change:

a. Predicted risks and mitigation actions taken in each case:

- Not filling the knowledge gap. If the knowledge gap has not been effectively closed, teams are not self-sufficient to keep the transformation after the change process is ended (Wong et al., 2005).

- Lack or infectivity of communication. At this step of the project there is a risk of declaring victory too soon. Communication needs to acknowledge the wins so far but without reducing the motivation for the changes that are still to come (Kotter, 2006). The guiding team decided to start a new fortnightly communication of results obtained by any market (both good and bad results) with the purpose of showing that the initiative was still running and also to let all know the achievements periodically. This communication was detected to have positive impact on the change efforts and more local markets started adopting the new decision-making model.

- Failure to cope with people's resistance to change. At this point, passive or covert resistance to change can drive the final result of the change effort (Bovey \& Hede, 2001). Even passive organizational 
cynicism, that can be powerless at the beginning of the initiative, could mean important setbacks at this point (Attaran, 2000; Imran et al., 2016).

b. Detected risks:

- As predicted, the risk of 'not filling the knowledge gap' started to have some effects on the results and to represent a clear risk for the success of the transformation. At this step of the process there was a notable difference between totally autonomous teams and the ones still needing a lot of support to perform website experiments. The excess of non-autonomous teams could produce the collapse of the guiding coalition not being able to give support to every team requiring assistance. Also, the guiding team was not able to keep encouraging new local teams to the initiative, so the initiative was not expanding as expected.

- Moreover, as predicted, the risk of 'failing to cope with people's resistance to change' started to have an impact on the change result. At this step of the process, there was a peak of work (not related with the change initiative) affecting all the teams involved in the initiative. The most active and autonomous teams continued experimenting on their websites, also, other non-autonomous teams continued experimenting with the support of the guiding coalition. However, some teams that had not previously shown any interest nor rejection for the initiative started trying to proceed in the old decision-making model (intuition driven, without experiments).

Step 8. Institutionalize new approaches: At this step, there are not main risks predicted or detected because it is an ongoing step that can take years.

Finally, some non-predicted and general risks for the success of the organizational change initiative were detected:

a. On the one hand, as general risks we should comment specially communication and knowledge gap, both risks were thoughtfully taken care of during the whole process, however the disparate set of employees involved in the initiative made it difficult to cope with everyone's communication and training needs. The communication, based on simplifying as much as possible the vision of the initiative, in order to engage as much employees as possible, was found to be too simplistic to be relevant for some local teams. And, after some general communications started not paying attention to them. The training documentation had a similar effect, while it was useful for some teams, that quickly started experimenting autonomously, other teams are still not able to experiment without support.

b. On the other hand, some other risks that are not so extensively commented in the literature were detected.

- First, as far as there were no key performance indicators designed to assess the results of the transformation, it is not possible to evaluate objectively the result of the organizational change initiative (Soja, 2016). This lack of evaluation might increase the risk of losing management support or team members motivation (Fui-Hoon Nah et al., 2001).

- Second, although it is not widely commented in the literature, there are some authors that warn of the need of providing anchoring points during the change process (Appelbaum et al., 2018; Kotter, 2006). After the sudden resistance to change that appeared during step 7 of the process, the lack of anchoring points was found to risk the global success of the change efforts.

- Third, because of the focus on the transformation process, the steps to follow and the potential risks of each stage, the original vision of the project was sometimes lost both for the guiding coalition and for the local teams, this might have increased the risk of losing team members motivation or sense of urgency (Ouedraogo \& Ouakouak, 2018).

- Finally, some of the teams were trying to use the new procedures as a cover to keep their old practices, this is, some teams were designing the experiments to get the results that they needed in order to validate their opinions. With it, those teams had the data to justify the decisions that were actually intuition-driven. This was not the purpose of the change initiative, as far as the data-driven 
decision-making culture should be fair in letting the user and the data guide the decisions and not the intuition guide the data.

Table 3 summarizes the results obtained, including the predicted and the detected risks for each step of the followed model.

\begin{tabular}{|l|c|c|c|c|c|c|c|c|c|}
\hline \multicolumn{1}{|c|}{ Risks Process } & Step 0 & Step 1 & Step 2 & Step 3 & Step 4 & Step 5 & Step 6 & Step 7 & General \\
\hline $\begin{array}{l}\text { 1. Not following an } \\
\text { organizational change procedure }\end{array}$ & $\mathrm{P}$ & & & & & & & & \\
\hline 2. Not filling the knowledge gap & & & & & $\mathrm{P}$ & $\mathrm{P}$ & & $\mathrm{P} \mathrm{X}$ & $\mathrm{X}$ \\
\hline $\begin{array}{l}\text { 3. Failure to cope with people's } \\
\text { resistance to change }\end{array}$ & & & & $\mathrm{P}$ & $\mathrm{X}$ & $\mathrm{P}$ & & $\mathrm{PX}$ & \\
\hline 4. Lack of sense of urgency & & $\mathrm{P}$ & & & & $\mathrm{X}$ & $\mathrm{P}$ & & \\
\hline $\begin{array}{l}\text { 5. Insufficient organizational } \\
\text { readiness for change }\end{array}$ & $\mathrm{P}$ & & $\mathrm{P}$ & & & & & & \\
\hline $\begin{array}{l}\text { 6.Insufficient management } \\
\text { support and involvement }\end{array}$ & & & $\mathrm{P}$ & & & & $\mathrm{X}$ & & \\
\hline $\begin{array}{l}\text { 7. Lack or infectivity of } \\
\text { communication }\end{array}$ & & & & & $\mathrm{P}$ & & $\mathrm{P}$ & $\mathrm{P}$ & $\mathrm{X}$ \\
\hline Other & & & & & & & & & $\mathrm{X}$ \\
\hline
\end{tabular}

$\mathrm{P}=$ Predicted risk; $\mathrm{X}=$ Detected risk

Table 3. Summary of risks predicted and detected in each step of the change process

As seen in the results, even if all the failure factors obtained from the literature were predicted to be a risk and most of them were detected as a risk during the project, they were found to only have moderate negative effect on the change result. A possible reason for that is the awareness of this risks beforehand. The fact that the whole planning of the organizational change was made taking into account the possible emergence of those seven risks and actions to mitigate some of them were outlines before the starting of the project, might have had an effect on the global risk of change failure. This is consistent with the first failure cause commented from the literature (Not following an organizational change procedure). According to previous literature, not only to follow an organizational change procedure, but also to be aware of and predict in advance the potential risks for the transformation (and setting mitigations actions accordingly when applicable) seem to play a role in the results (Hamdi \& Abouabdellah, 2018).

Moreover, not action was taken to mitigate some of the predicted risks, regardless of the importance given to those risks in the literature (i.e. insufficient readiness for change). However, those predicted risks showed no detectable risk for the success of the organizational change effort. This might be due to the interrelated effects of most of the failure factors, being ones found to be moderators of others' effect (Aslam et al., 2018; Ouedraogo \& Ouakouak, 2018). With it, acting to mitigate some risks might be more efficient than acting against others. Therefore, future research could focus on the relationships between failure factors and the key mitigation actions to be performed in order to effectively decrease the risk of negative results for the organizational change process.

According to the literature, all the seven studied factors were predicted to be a risk for the transformation and most of them were actually detected as risks during the project. Nevertheless, no risk was detected until the second half of the project (measured in steps). A possible reason for that is that the risks where present from the beginning of the process, however they were not detected until they were notable enough to not to be negligible because of the lack of ability to diagnose (Long et al., 2018). If the detection of actual risks was made from the beginning of their emergence, they might have been easier to mitigate. Furthermore, even if the company was aware of the risks to focus attention on, they had not clear detection processes nor mitigation actions, what is called in the literature. 
Hence, we suggest that future research could address not only the risks for transformation success but on how to detect those risks and how to deal with them once detected.

Finally, accordingly to the literature, an organizational change process is shown to a highly context dependent process (Imran et al., 2016; Jansson, 2013). Not only the specific organization or the teams involved are going to affect the results of the transformation, but also the specific period and the rest of the projects affecting to the same team members.

\section{Conclusions}

Data-driven decision-making is a growing trend that lots of companies are nowadays willing to adopt. However, the organizational transformation needed is not always as simple and logical as it could seem and the comfort of the old habits can dim the change effort. In this study, the potential problems that may arise in a real company's transformation (from a traditional intuition-driven decision-making model to a data-driven model) once the company has been forewarned of the main failure factors have been identified by means of a case study. With it, this study contributes to both theory and practice by offering a real vision of how companies address this kind of digital transformations. On the one hand this study shows how some widely studied failure factors for organizational change would not represent such a high risk if the companies were aware of them. On the other hand, practitioners might take the identified failure factors into account and consider them as potential risks for their organizational transformations in their strategic planning when considering making the transition towards data-driven companies. Finally, this study adds to the existing literature and opens up new avenues for further research in the area of organizational change.

The analyzed organization for this case study is the digital platforms team of an automotive brand. While being a digital team related to software products, the industrial organizational context of this department has played a key role in the past processes and decision-making procedures for the development of the website.

In the paper, a literature review on the organizational change models has been presented, and seven of the most commented causes for organizational change failure in the literature have been discussed (not following an organizational change procedure, not filling the knowledge gap, failure to cope with people's resistance to change, lack of sense of urgency, insufficient organizational readiness for change, insufficient management support and involvement and lack or infectivity of communication). The case study has been analyzed with special focus on detecting these risks and how the company acted to prevent or mitigate them. However, most of the commented risks were anyway detected within the transformation.

Although the company proved having a solid plan for the organizational transformation, they found some problems determining the specific risks to be aware of in each step of the process as far as the specific actions to plan in order to mitigate them. There is abundant literature on success and failure causes of organizational change but mapping those causes within the process is not an easy task for practitioners.

As a case study research approach, the major limitation of this paper is that it may not offer a generalized picture of the phenomenon. Moreover, considering the high contextual dependence of organizational transformations, the results of this study are by no means meant to be taken as a closed final conclusion. Therefore, future work could be conducted in similar case companies (digitalization transformations within traditional industrial companies) in order to validate the results and further explain the potential problems that may fall down this kind of change initiatives. Also, future work on the topic of organizational change could address the specific problems and their specific alleviation measures for each phase of a transformation process.

\section{Declaration of Conflicting Interests}

The authors declared no potential conflicts of interest with respect to the research, authorship, and/or publication of this article. 


\section{Funding}

This work was supported in part under the Industrial Doctorate Grant DI 052/2016 (Secretaria d'Universitats $i$ Recerca, Generalitat de Catalunya).

\section{References}

Ainul-Azyan, Z.H., Pulakanam, V., \& Pons, D. (2017). Success factors and barriers to implementing lean in the printing industry: A case study and theoretical framework. Journal of Manufacturing Technology Management, 28(4), 458-484. https://doi.org/10.1108/JMTM-05-2016-0067

Alsulami, M., Rahim, M., \& Scheepers, H. (2013). Development of a model to understand how consultants manage conflicts during ERP post-implementation change process: A dialectic perspective. Proceedings of the 24th Australasian Conference on Information Systems (July 2014).

Antony, J., \& Banuelas, R. (2002). Key ingredients for the effective implementation of Six Sigma program. Measuring Business Excellence, 6(4), 20-27. https://doi.org/10.1108/13683040210451679

Appelbaum, S.H., Cameron, A., Ensink, F., Hazarika, J., Attir, R., Ezzedine, R., et al. (2017). Factors that impact the success of an organizational change: a case study analysis. Industrial and Commercial Training, 49(5), 213-230. https://doi.org/10.1108/ICT-02-2017-0006

Appelbaum, S.H., Profka, E., Depta, A.M., \& Petrynski, B. (2018). Impact of business model change on organizational success. Industrial and Commercial Training, 50(2), 41-54. https://doi.org/10.1108/ICT-07-2017-0058

Aslam, U., Muqadas, F., Imran, M.K., \& Saboor, A. (2018). Emerging organizational parameters and their roles in implementation of organizational change. Journal of Organizational Change Management, 31(5), 1084-1104. https://doi.org/10.1108/JOCM-08-2017-0300

Attaran, M. (2000). Why does reengineering fail? A practical guide for successful implementation. Journal of Management Development, 19(9), 794-801. https://doi.org/10.1108/02621710010378237

Baxter, P., \& Jack, S. (2008). Qualitative Case Study Methodology: Study Design and Implementation for Novice Researchers. The Qualitative Report, 13(4), 544-559. Available at: https://nsuworks.nova.edu/tqr/vol13/iss4/2

Bovey, W.H., \& Hede, A. (2001). Resistance to organisational change: The role of defence mechanisms. Journal of Managerial Psychology, 16(7), 534-548. https://doi.org/10.1108/EUM0000000006166

Brisson-Banks, C.V. (2010). Managing change and transitions: A comparison of different models and their commonalities. Library Management, 31(4), 241-252. https://doi.org/10.1108/01435121011046317

Bullock, R.J., \& Batten, D. (1985). It's Just a Phase We're Going Through: A Review and Synthesis of OD Phase Analysis. Group \& Organization Management, 10(4), 383-412. https://doi.org/10.1177/105960118501000403

Cândido, C.J.F., \& Santos, S.P. (2015). Strategy implementation: What is the failure rate? Journal of Management and Organization, 21(2), 237-262. https://doi.org/10.1017/jmo.2014.77

Cao, G., Clarke, S., \& Lehaney, B. (2000). A systemic view of organisational change and TQM. TQM Magazine, 12(3), 186-193. https://doi.org/10.1108/09544780010320241

Christopher, W.F. (2011). A new management for enduring company success. Kybernetes, 40(3/4), 369-393. https://doi.org/10.1108/03684921111133638

Chrusciel, D., \& Field, D.W. (2006). Success factors in dealing with significant change in an organization. Business Process Management Journal, 12(4), 503-516. https://doi.org/10.1108/14637150610678096

Condo, C., \& Seguin, B. (2019). AD\&D Pros: Stop Guessing And Start Experimenting.

Cray, P. (2014). The digital transformation of business. Boston, MA: Harvard Business Publishing. https://doi.org/10.15547/tjs.2017.s.01.065 
Crook, T., Frasca, B., Kohavi, R., \& Longbotham, R. (2009). Seven pitfalls to avoid when running controlled experiments on the web. Proceedings of the 15th ACM SIGKDD International Conference on Knowledge Discovery and Data Mining - KDD '09 (1105). https://doi.org/10.1145/1557019.1557139

Elrod, P.D.I., \& Tippett, D.D. (2002). The death valley of change. Journal of Organizational Change Management, 15(3), 273-291. https://doi.org/10.1108/09534810210429309

Erwin, D.G., \& Garman, A.N. (2010). Resistance to organizational change: linking research and practice. Leadership \& Organization Development, 31(1), 39-56. https://doi.org/10.1108/01437731011010371

Fabijan, A., Dmitriev, P., Holmstrom-Olsson, H., \& Bosch, J. (2018). The Online Controlled Experiment Lifecycle. IEEE Software, 1, 1. https://doi.org/10.1109/MS.2018.2875842

Fabijan, A., Dmitriev, P., McFarland, C., Vermeer, L., Holmström Olsson, H., \& Bosch, J. (2018). Experimentation growth: Evolving trustworthy A/B testing capabilities in online software companies. Journal of Software: Evolution and Process, (December 2017), e2113. https://doi.org/10.1002/smr.2113

Fabijan, A., Dmitriev, P., Olsson, H. H., \& Bosch, J. (2017). The Evolution of Continuous Experimentation in Software Product Development: From Data to a Data-Driven Organization at Scale. In Proceedings - 2017 IEEE/ACM 39th International Conference on Software Engineering, ICSE 2017 (770-780). https://doi.org/10.1109/ICSE.2017.76

Fui-Hoon Nah, F., Lee-Shang Lau, J., \& Kuang, J. (2001). Critical factors for successful implementation of enterprise systems. Business Process Management Journal, 7(3), 285-296. https://doi.org/10.1108/14637150110392782

Galli, B.J. (2019). An Engineering Manager's Guide for Commonly Used Change Management Approaches. IEEE Engineering Management Review, 47(3), 118-126. https:/ / doi.org/10.1109/EMR.2019.2896301

Hamdi, S. El, \& Abouabdellah, A. (2018). Literature review of implementation of an entreprise resources planning: Dimensional approach. In Proceedings - GOL 2018: 4th IEEE International Conference on Logistics Operations Management (1-7). IEEE. https://doi.org/10.1109/GOL.2018.8378095

Hughes, M. (2011). Do 70 Per Cent of All Organizational Change Initiatives Really Fail? Journal of Change Management, 11(4), 451-464. https://doi.org/10.1080/14697017.2011.630506

Imran, M.K., Rehman, C.A., Aslam, U., \& Bilal, A.R. (2016). What's organization knowledge management strategy for successful change implementation? Journal of Organizational Change Management, 29(7), 1097-1117. https://doi.org/10.1108/JOCM-07-2015-0130

Jansson, N. (2013). Organizational change as practice: A critical analysis. Journal of Organizational Change Management, 26(6), 1003-1019. https://doi.org/10.1108/JOCM-09-2012-0152

Johansson, C., \& Heide, M. (2008). Speaking of change: three communication approaches in studies of organizational change. Corporate Communications: An International Journal, 13(3), 288-305.

https://doi.org/10.1108/CCIJ-02-2015-0009

Kanter, R.M., Stein, B., \& Jick, T.D. (1992). The Challenge of Organizational Change: How Companies Experience It and Leaders Guide It. New York.

Kaushik, A. (2006). Experimentation and Testing: A Primer. Available at: https://www.kaushik.net/avinash/experimentation-and-testing-a-primer/ (Accessed: March 2019).

Kohavi, R., Deng, A., Longbotham, R., \& Xu, Y. (2014). Seven rules of thumb for web site experimenters. Proceedings of the 20th ACM SIGKDD International Conference on Knowledge Discovery and Data Mining - KDD '14, (1857-1866). https://doi.org/10.1145/2623330.2623341

Kohavi, R., Henne, R.M., \& Sommerfield, D. (2007). Practical Guide to Controlled Experiments on the Web: Listen to Your Customers not to the HiPPO. In Proceedings of the 13th ACM SIGKDD international conference on Knowledge Discovery and Data Mining, (959-967). San Jose, CA, United States. https://doi.org/10.1145/1281192.1281295 
Kohnke, O. (2016). It's not just about technology: The people side of digitalization. In Shaping the Digital Enterprise: Trends and Use Cases in Digital Innovation and Transformation (69-91). https://doi.org/10.1007/978-3-319-40967-2

Kotter, J., \& Rathgeber, H. (2005). Our Iceberg is melting: Changing and succeeding under any condition.

Kotter, J.P. (1996). Leading Change. Boston: Harvard Business School Press.

Kotter, J.P. (2006). Leading change - why transformation efforts fail. Harvard Business Review, Best if HB (Januar). Available at: http://www.institute.nhs.uk/nhs alert/article of the month/april 2011 article of the month.html

Lewin, K. (1951). Field Theory of Social Science: Selected Thoretical Papers. New York: D. Cartwright, Ed., Harper \& B.

Long, T.B., Looijen, A., \& Blok, V. (2018). Critical success factors for the transition to business models for sustainability in the food and beverage industry in the Netherlands. Journal of Cleaner Production, 175, 82-95. https://doi.org/10.1016/j.jclepro.2017.11.067

Martin, A.J., Jones, E.S., \& Callan, V.J. (2005). The role of psychological climate in facilitating employee adjustment during organizational change. European Journal of Work and Organizational Psychology, 14(3), 263-289. https://doi.org/10.1080/13594320500141228

Montgomery, D.C. (2001). Design and Analysis of Experiments. Design and Analysis of Experiments. New York: John Wiley. https://doi.org/10.1198/tech.2006.s372

Neely, S., \& Stolt, S. (2013). Continuous delivery? Easy! Just change everything (well, maybe it is not that easy). In Proceedings - AGILE 2013 (121-128). https://doi.org/10.1109/AGILE.2013.17

Olsson, H.H., \& Bosch, J. (2014). From Opinions to Data-Driven Software R\&D: A Multi-Case Study On How To Close The “Open Loop" Problem. Continuous software engineering, 155-164. https://doi.org/10.1109/SEAA.2014.75

Olsson, H.H., Bosch, J., \& Fabijan, A. (2017). Experimentation that Matters: A Multi-case Study on the Challenges with A/B Testing. 8th International Conference on Software Business, 304(June), 179-185.

https://doi.org/10.1007/978-3-642-30746-1

Ouedraogo, N., \& Ouakouak, M.L. (2018). Impacts of personal trust, communication, and affective commitment on change success. Journal of Organizational Change Management, 31(3), 676-696. Retrieved from https://doi.org/10.5465/AMBPP.2015.12809abstract

Piderit, S.K. (2000). Rethinking resistance and recognizing ambivalence: A multidimensional view of attitudes toward an organizational change. Academy of Management Review, 25(4), 783-794.

https://doi.org/10.5465/AMR.2000.3707722

Rajan, R., \& Ganesan, R. (2017). A critical analysis of John P. Kotter's change management framework. Asian Journal of Research in Business Economics and Management, 7(7), 181. https://doi.org/10.5958/2249-7307.2017.00106.2

Rashford, N.S., \& Coghlan, D. (1989). Phases and Levels of Organizational Change. Journal of Managerial Psychology, 4(2), 17-22.

Rissanen, O., \& Munch, J. (2015). Continuous Experimentation in the B2B Domain: A Case Study. In Proceedings 2nd International Workshop on Rapid Continuous Software Engineering, RCoSE 2015, 12-18.

https://doi.org/10.1109/RCoSE.2015.10

Soja, P. (2016). Reexamining Critical Success Factors for Enterprise System Adoption in Transition Economies: Learning from Polish Adopters. Information Technology for Development, 22(2), 279-305.

https://doi.org/10.1080/02681102.2015.1075189

Spear, S.J. (2004). Learning to Lead at Toyota. Harvard Business Review, 82, 78-86+151. https://doi.org/10.1225/2890

Tang, D., Agarwal, A., O’Brien, D., \& Meyer, M. (2010). Overlapping Experiment Infrastructure: More, Better, Faster Experimentation. In Proceedings of the 16th ACM SIGKDD International Conference on Knowledge Discovery and Data Mining - KDD '10 (17). https://doi.org/10.1145/1835804.1835810 
Vakola, M., \& Wilson, I.E. (2004). The challenge of virtual organisation: critical success factors in dealing with constant change. Team Performance Management: An International Journal, 10(5), 112-120.

https://doi.org/10.1108/13527590410556836

Venugopal, C., \& Suryaprakasa-Rao, K. (2011). Learning from a failed ERP implementation: a case study research. International Journal of Managing Projects in Business, 4(4), 596-615. https:/ /doi.org/10.1108/17538371111164038

Wassner, H., \& Brebion, A. (2018). Demystifying A/B Testing Statistics. AB Tasty Whitepaper.

Wong, A., Chau, P.Y.K., Scarbrough, H., \& Davison, R. (2005). Critical failure factors in ERP implementation. 9th Pacific Asia Conference on Information Systems: I.T. and Value Creation, PACIS 2005.

Yin, R.K. (2002). Applications of case study research. Applied Social Research Methods Series. Robert K. Yin Ed.

Yin, R.K. (2003). Case Study Research: Design and Methods. Robert K. Yin Ed.

Journal of Industrial Engineering and Management, 2020 (www.jiem.org)

\section{(c) (i) (8)}

Article's contents are provided on an Attribution-Non Commercial 4.0 Creative commons International License. Readers are allowed to copy, distribute and communicate article's contents, provided the author's and Journal of Industrial Engineering and Management's names are included. It must not be used for commercial purposes. To see the complete license contents, please visit https://creativecommons.org/licenses/by-nc/4.0/. 